\title{
Teoria do Flow e a Autoeficácia: contribuições para o processo de ensino e aprendizagem da matemática
}

\author{
Hermes Renato Hildebrand - PUC/SP \\ Ana Maria Antunes de Campos - PUC/SP
}

\section{Resumo}

As dificuldades das pessoas para aprender Matemática, por vezes, é atribuída aos métodos de ensino, ao tipo de currículo, aos fatores intrínsecos aos estudantes e formação dos professores. Vários pesquisadores têm se debruçado sobre o assunto a fim de compreender as características que embasam esse tipo de cognição. Entre as diversas situações abordadas pelos educadores identifica-se aspectos como a afetividade, autoeficácia e a cognição humana, interferindo na aprendizagem de matemática. Neste sentido, este ensaio, busca problematizar sobre o conceito de Flow elaborado por Mihaly Csikszentmihalyi e a Teoria Social Cognitiva de Albert Bandura. Aqui, busca-se verificar como esses conceitos podem facilitar o processo de cognição matemática. Os resultados dessa pesquisa revelam que as atividades e tarefas matemáticas desenvolvidas com base na Metodologia Ativa, transformam-se positivamente quando atingimos o estado mental de Flow e atuamos com autoeficácia possibilitando a imersão, concentração e atenção focada.

Palavras-chave: Aprendizagem Matemática, Metodologia Ativa, Flow, Autoeficácia e Jogos.

* Professor, Doutor e Vice-Coordenador do Programa de Estudos Pós-Graduados em Tecnologia da Inteligência e Design Digital da Pontifícia Universidade Católica de São Paulo - PUC/SP. E-mail: hrenatoh@pucsp.com e hrenatoh@gmail.com.

** Doutoranda no Programa de Estudos Pós-Graduados em Educação Matemática da Pontifícia Universidade Católica de São Paulo - PUC/SP. E-mail: camp.ana@hotmail.com 


\section{falta título em inglês}

\section{Abstract}

People's difficulties in learning mathematics are sometimes attributed to teaching methods, the type of curriculum, factors intrinsic to students and teacher training. Several researchers have studied the subject to understand the characteristics that underlie this type of cognition. Among the various situations addressed by educators, aspects such as affectivity, self-efficacy and human cognition are identified, interfering in the learning of mathematics. In this sense, this essay seeks to problematize the concept of Flow developed by Mihaly Csikszentmihalyi and Albert Bandura's Social Cognitive Theory. Here, we seek to verify how these concepts can facilitate the mathematical cognition process. The results of this research reveal that the activities and mathematical tasks developed based on the Active Methodology, transform positively when we reach the mental state of Flow and act with self-efficacy allowing immersion, concentration, and focused attention.

Keywords: Mathematical Learning, Active Methodology, Flow, Self-efficacy and Games.

\section{falta título em espanhol}

\section{Resumen}

Las dificultades de las personas para aprender matemáticas a veces se atribuyen a los métodos de enseñanza, el tipo de plan de estudios, los factores intrínsecos a los estudiantes y la formación de los profesores. Varios investigadores han estudiado el tema para comprender las características que subyacen a este tipo de cognición. Entre las diversas situaciones abordadas por los educadores, se identifican aspectos como la afectividad, la autoeficacia y la cognición humana, interfiriendo en el aprendizaje de las matemáticas. En este sentido, este ensayo busca problematizar el concepto de Flow desarrollado por Mihaly Csikszentmihalyi y la Teoría Cognitiva Social de Albert Bandura. Aquí, buscamos verificar cómo estos conceptos pueden facilitar el proceso de cognición matemática. Los resultados de esta investigación revelan que las actividades y tareas matemáticas desarrolladas en base a la Metodología Activa, se transforman positivamente cuando llegamos al estado mental de Flow y actuamos con autoeficacia permitiendo la inmersión, concentración y atención enfocada.

Palabras clave: aprendizaje matemático, metodología activa, fluidez, autoeficacia y juegos. 


\section{Introdução}

A Matemática sempre gerou questionamentos acerca das dificuldades de aprendizagem que, por vezes, é atribuída aos métodos de ensino, aos padrões lógicos do conhecimento, aos fatores intrínsecos aos estudantes e formação dos professores. Nesse sentido, alguns pesquisadores (SANTOS et al., 2012; BARBOSA, 2015; ANDRADE; PRADO; CARMO, 2015) têm se debruçado sobre o assunto com a finalidade de compreender as características e os elementos que interferem no processo de cognição da matemática e da lógica.

Nesse sentido, este artigo busca responder questões que envolvem estados mentais nos quais o aprendizado se desenvolve de modo mais intenso, possibilitando o desenvolvimento por meio da autoeficácia. O método de aprendizagem utilizado nesta reflexão é centrado na ação que transforma a cognição tornando-a mais efetiva. Assim, para essa reflexão, partimos da seguinte questão: o estado mental de Flow, de Mihaly Csikszentmihalyi, e a Teoria Social Cognitiva de Albert Bandura, podem beneficiar o processo de aprendizagem da matemática?

\section{A Teoria do Flow}

A Teoria do Flow foi estruturada a partir da década de 1970 com estudos do psicólogo húngaro Mihaly Csikszentmihalyi que ficou impressionado com o fato de que pessoas que se envolvem profundamente com uma atividade, podem desconsiderar necessidades básicas como a fome, a fadiga e algum tipo de desconforto. Assim, ele formulou uma teoria que teve origem no desejo de se compreender o quanto uma atividade pode ser motivadora por si só, independentemente de gerar algum produto ou de produzir qualquer bem extrínseco.

Csikszentmihalyi (1990) investiga a natureza e as condições de felicidade de se jogar xadrez, de se fazer alpinismo e de se dançar, e de outras atividades que enfatizam o prazer como a principal razão para sua execução. Para descrever essas situações que envol- 
vem a sensação de alegria e o profundo prazer, o pesquisador usa o termo "experiência ideal". Esse é um momento relaxante onde mente e corpo ultrapassam seus limites e realizam atividades que exigem esforços voluntários tornando algo difícil de ser realizado em algo que vale a pena. (Csikszentmihalyi; Nakamura, 2002).

Segundo o autor, todas as experiências humanas são representadas na mente como informação e a "experiência ideal" resulta de uma estruturação na qual há uma ordem da consciência. Elas emergem de situações nas quais focamos metas realistas a partir de nossas habilidades e quando concentramos nossa atenção em uma determinada tarefa e esquecemos tudo ao nosso redor (Csikszentmihalyi, 1990), independente das circunstâncias externas e do ambiente social em que estamos inseridos. É possível aprender a encontrar prazer e propósito satisfatório na vida, nas experiências e nas atividades vivenciadas. Assim, nas palavras do autor:

O nosso nível de felicidade, em última análise, depende de como nossos filtros mentais interpretam experiências cotidianas. Felicidade depende da harmonia interior, e não da nossa capacidade de exercer controle sobre as grandes forças do universo. Há pessoas que, independentemente das suas condições materiais estão satisfeitos e têm uma maneira de fazer com que aqueles, em seu entorno, sejam mais felizes. Por outro lado, há pessoas que apesar de ter sido abençoado com tanto dinheiro e poder, são infelizes. (CSIKSZENTMIHALYI, 1990, p.1).

Para esse pesquisador, no instante em que uma pessoa está totalmente concentrada e imersa em uma atividade que ela tem prazer em realizar, atinge-se um estado mental de obtenção de fluxo, isto é, um sentimento de satisfação e sucesso ao se desenvolver essa atividade. Csikszentmihalyi define o Estado de Flow como "um estado em que as pessoas estão tão envolvidas em uma atividade que nada mais parece importar; a experiência é tão agradável que as pessoas continuarão a fazê-lo mesmo a um custo elevado, pelo simples fato de fazê-lo" (1990, p. 4).

Mihaly identifica a presença de oito elementos que determinam esse estado mental de Flow e, para tanto, precisamos ter: 
1. Metas claras e feedback imediato: deve-se ter conhecimento preciso das tarefas que devem ser realizadas e, a todo o instante deve-se receber feedback das ações que estão sendo realizadas para se conseguir atingir as metas estabelecidas.

2. Equilíbrio entre oportunidade da ação e capacidade de realizá-las: deve-se saber que somos capazes de fazer determinadas atividades e perceber que possuímos plena habilidade para superar os desafios propostos.

3. Sensação de controle: deve-se ter a habilidade de controlar o próprio desempenho e as ações serão todas direcionadas para a atividade.

4. Concentração profunda na ação que se funde com a consciência: deve-se ter concentração total da atenção na atividade.

5. Foco temporal no presente: abandona-se os elementos sobre o passado e o futuro. A atenção deve estar focada no presente. A consciência não tem espaço para fatos do passado ou do futuro.

6. A noção de tempo é alterada: tem-se a percepção de que o tempo não passa, ou passa mais rápido, ou ainda passa mais lento. Isto é, a noção de tempo deixa de existir.

7. Perda da autoconsciência reflexiva e transcendência das fronteiras do self: identifica-se a suspensão temporária da consciência de si mesmo, isso pode conduzir a autotranscedência.

8. Por fim, a expectativa se torna autotélica: a atividade é tão gratificante que o objetivo final passa a ter uma única justificativa do processo. É algo que não possui propósito ou finalidade além de si mesmo.

Csikszentmihalyi (1999) defende que as atividades como: artes, atividades físicas esportivas, jogos e outras atividades humanas que promovem a criatividade, possibilitam a diversão, o envolvimento e o entretenimento, e podem ser utilizadas como ponto de 
partida para desenvolver a concentração, o interesse, a motivação, o lúdico, favorecendo um profundo aprendizado.

As atividades que possibilitam atingir o estado de Flow, proporcionam o equilíbrio entre a capacidade e o desafio, a pessoa tem clareza dos objetivos a serem atingidos, a noção de tempo é modificada, a sensação de controle é alterada, e a pessoa consegue controlar suas ações e o ambiente. Segundo Csikszentmihalyi (1999), é por essas razões que é fácil entrar no estado de Flow, principalmente, por meio dos jogos.

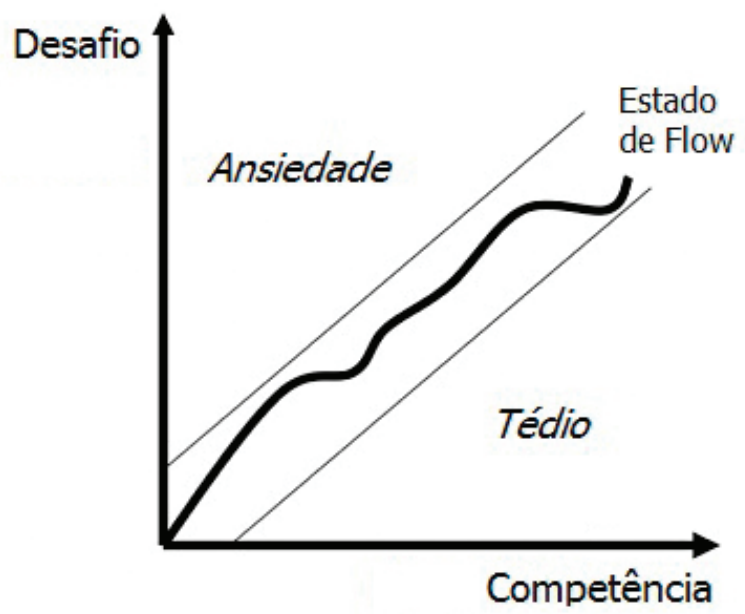

Figura 1 - Esquema do estado de Flow

Fonte: (CSIKSZENTMIHALYI, 1990, p. 74)

A Teoria do Flow permite analisar a intensidade do desafio e o quanto de habilidades que uma pessoa precisa para experimentar ou vivenciar uma situação. E, para atingir o Flow, é necessário que os desafios e habilidades estejam acima da média. Isso acontece quando se está realizando uma atividade que realmente se gosta do que se está fazendo e, assim, temos uma sensação de prazer.

Ao atingir o estado mental total de concentração: no estado de Flow, as pessoas vivenciam situações prazerosas, nas quais verifi- 
cam uma imensa concentração, total interesse, motivação, criatividade e, consequentemente, acontece o aprendizado. Nesta direção, vamos encontrar a Teoria do Flow agindo em diversas áreas do conhecimento, em especial na educação matemática. As experiências do Flow transformam a nossa motivação, interação e criatividade, permitindo o desenvolvimento de habilidades necessárias para se aprender algo. Neste estado mental de concentração não ocorre a ansiedade (que é sentida quando o desafio é maior do que a nossa competência) e nem o tédio (quando a competência é maior do que o desafio).

\section{Teoria Social Cognitiva}

Teoria Social Cognitiva foi desenvolvida por Albert Bandura (1999) e apresenta os mecanismos e efeitos das crenças na autoeficácia e, assim, fornece elementos sobre como melhor desenvolver habilidades que permitam que as pessoas sejam capazes de aplicá-las perante situações de dificuldades. Mais especificamente, Bandura procura discutir o papel da autoeficácia no contexto do ensino e aprendizagem.

A autoeficácia foi definida por Bandura como a crença "na capacidade de organizar e executar os cursos de ação necessários para produzir determinadas realizações" (1997, p. 3).

A autoeficácia matemática pode ser definida como uma avaliação situacional específica de um problema no qual um indivíduo tem total confiança em sua capacidade de realizar, com sucesso, uma determinada tarefa, neste caso estamos tratando da resolução de um problema matemático. ${ }^{1}$

Desse modo, associado ao estado mental de Flow vamos observar a autoeficácia como, um estado psicológico, no qual a pessoa tem a convicção de que é capaz de realizar uma determinada tarefa.

Mathematics self-efficacy can be distinguished from other measures of attitudes toward mathematics in that mathematics self-efficacy is a situational or problem-specific assessment of an individual's confidence in her or his ability to success-fully perform or accomplish a particular task or problem. (HACKET'T; BETZ, 1989, p. 262. Tradução nossa). 
Esse conceito gerado a partir da autoeficácia envolve o modo de se "aprender a aprender". Também envolve a crença de que, com empenho, podemos administrar os acontecimentos ao nosso redor, gerando os efeitos desejados.

A autoeficácia está associada ao desafio e ao estado de Flow que pode ser aplicado ao contexto de resolução de problemas, particularmente, os de matemática. Além disso, a relação entre a autoeficácia e as experiências do fluxo estão relacionadas aos dois conceitos que determinam o Flow: desafio e competência. Identificar se algo é tão difícil de ser resolvido nos conduz a observar a ansiedade e o tédio como elementos centrais ao estado mental do Flow. A aprendizagem matemática continua sendo uma temática muito interessante a ser pesquisada, visto que é uma questão que ainda permanece sem solução.

De acordo com Geist (2010), os alunos em geral são atormentados pela ansiedade de aprender matemática e, assim, desenvolvem atitudes negativas em direção ao conhecimento dessa ciência. Estudos indicam que a ansiedade matemática geralmente está associada à falta de confiança de se tratar com os conceitos lógicos que envolvem as situações matemáticas (Stuart, 2000; Gourgey, 1984). De acordo com Acelajado (2004) a ansiedade matemática também está associada a uma perda de autoestima quando o aprendiz se depara com a situação matemática. Scarpello (2007) argumenta que a ansiedade matemática pode ser causada por experiências negativas anteriores "em sala de aula, influenciadas pelos pais e lembrando-se de um fraco desempenho em matemática” (p. 34). Desse modo, repensar os métodos de ensino com os pais e professores pode ajudar no processo de aprendizagem e é um dos caminhos para modificar esse cenário. Os estudos acerca da Metodologia Ativa podem amparar nessas discussões.

\section{A Metodologia Ativa}

A Metodologia Ativa envolve o processo de aprendizagem pela ação que pode ser desenvolvido utilizando os conceitos de Flow 
e de autoeficácia melhorando o processo de ensino e aprendizagem e as práticas pedagógicas. Nesse contexto o aprendiz assume independência em seu processo de aprender, de fato, ele controla a forma de adquirir o seu conhecimento. A metodologia ativa consiste em uma série de técnicas, procedimentos e processos pelos quais o professor tem o papel de mediador entre o aprendiz e o conhecimento.

A metodologia ativa tem sido implementada a partir de diversas estratégias. As mais conhecidas são aprendizagem baseada em projetos (Project Based Learning - PBL); Aprendizagem por solução de caso (Teaching Case); a aprendizagem em equipe (Team Based Learning - TBL); e aprendizagem por meio dos jogos (Game Based Learning - GLD). A maior dificuldade na aplicação desses métodos é a adequação dos conteúdos curriculares ao nível de conhecimento e de interesse dos alunos. Por outro lado, também devemos observar que esses métodos são difíceis de serem aplicados para muitas pessoas, pois o processo de mediação ocorre de modo individualizado e a atenção deve ser dada a cada aluno.

Esse constructo determina que o foco do processo de ensino está no aprendiz e a aprendizagem é direcionada por descoberta, investigação ou na resolução de problemas. A Metodologia Ativa contrapõe-se ao método tradicional pois não está centrada no conhecimento do professor. John Dewey definiu que a prática educativa deveria estar baseada em um processo de busca do conhecimento pelo próprio aluno. Portanto, o papel do professor nessas estratégias é de oferecer possibilidades de ação que leve o aluno a pesquisar o conhecimento e desenvolver-se pela sua própria iniciativa, a partir de seu conhecimento e no seu tempo, isso é, o professor é apenas um mediador.

Uma das experiências que faz uso da Metodologia Ativa é a sala de aula invertida (flipped classroom) que é um modelo educacional, em que o aluno é o protagonista do seu aprendizado. Ele aprende de forma autônoma e pode utilizar o apoio das Tecnologias Digitais da Informação e da Comunicação (TDIC). Segundo José Armando Valente (2014), os formatos adotados para a Educação a Distância 
(EaD) variam desde o uso de Broadcast (é uma transmissão de informação por meio de uma mídia qualquer. Ela acontece via ondas de rádio, satélite, cabos, fibras ópticas, linhas telefônicas, internet etc.) e, nesse caso, não temos a interação mediada do professor-aluno. Já, do outro lado, temos o conhecimento mediado pelas TDIC que é uma abordagem da "escola virtual" denominada de "estar junto virtual” (Valente, 1998).

A sala de aula invertida é caracterizada como uma forma de e-learning, em que os conteúdos e as instruções sobre eles acontecem de maneira online e antes da aula presencial. As atividades são realizadas por meio de resolução de problemas e projetos, discussões em grupo e os conteúdos básicos são estudados antes da aula. Valente afirma que, "na educação a distância, no uso de software do tipo simulação, na construção de narrativas digitais, e na implantação da abordagem híbrida de ensino e de aprendizagem" (2014) temos a implantação da aprendizagem por meio da sala de aula invertida.

Concluindo, Pavanelo e Lima (2017), afirmam que

a sala de aula invertida possibilita o professor desenvolver atividades de aprendizagem interativa em grupo na sala de aula e orientações baseadas nas tecnologias digitais fora da sala de aula, tendo como característica marcante não utiliza o tempo em sala com aulas expositivas (p. 756).

Esses autores também indicam que a afetividade nas relações entre os professores e os alunos, a partir das práticas pedagógicas, favorecem as relações entre os sujeitos e os conteúdos acadêmicos que devem ser ministrados. Assim, a Metodologia Ativa adequada ao aprendiz, sem gerar ansiedade e tédio, possibilita a imersão do aluno num estado de Flow e coopera com a forma de se adquirir conhecimento por meio do "aprender a aprender", isto é, pela autoeficácia.

A Metodologia Ativa não é estática, visto que permite ao aprendiz explorar, discutir e refletir acerca do seu próprio processo de aprendizagem. Isso pode ser feito a partir da mediação e a todo o momento. Assim, o aprendiz age utilizando, plenamente, suas habilidades cognitivas, afetivas e emocionais. 
Por outro lado, temos o uso do jogo que não é, necessariamente, uma Metodologia Ativa, entretanto, pode ser utilizado como estratégia para promover a aprendizagem de forma ativa. Fundamentado na premissa de que o aprendiz, por meio dessa ferramenta, é instigado a deixar de ser mero receptor de informações e assumir sua posição enquanto aprendiz que pode desenvolver novas habilidades como: autonomia, iniciativa, autoeficácia, autoestima e criatividade.

Por meio das características do estado de Flow (metas e objetivos claros; feedback imediato sobre o progresso que está se realizando; equilíbrio entre a ação e a capacidade de realizá-la; sensação de controle sobre a atividade; concentração profunda; foco temporal; fim da noção de tempo real; perda da autoconsciência reflexiva e atividade gratificante) o professor desafia e estimula o aluno a realizar sua missão no jogo. O jogador/aprendiz vence cada desafio, desenvolve estratégias para explorar as situações problemas, faz conjecturas e utiliza de suas habilidades para realizar as atividades propostas.

\section{As atividades matemáticas encaradas como um jogo}

As pessoas utilizam suas percepções para a eficácia e para escolher o que fazer e decidir quanto esforço devem investir numa atividade que pretendem realizar. Acreditamos que os estudantes procuram atividades que satisfazem suas necessidades e buscam realizar tarefas de forma prazerosa. Contudo, a maior parte das atividades empregadas nas aulas de matemáticas são desagradáveis e às vezes muito desafiadoras, causando o tédio ou a ansiedade.

Encontrar o equilibrio entre os desafios e as habilidades dos aprendizes é um dos caminhos para a motivação e, consequentemente, para "aprender a aprender". Nesse sentido, para se adquirir o conhecimento matemático é fundamental promover atividades que permitam ao estudante atingir o estado mental de Flow.

Essas atividades devem permitir o equilíbrio entre os desafios a serem atingidos e as habilidades dos estudantes. A integração desses dois fatores possibilitará que a ação e a consciência se fun- 
dam; a atenção será concentrada nos estímulos que são relevantes para atingir os objetivos; a concentração é exclusivamente sobre o que o estudante está fazendo naquele momento, excluindo os estímulos intrusivos que possam tirar o foco de sua atenção.

Segundo Csikszentmihalyi (2014) o jogo é uma das atividades que possibilita a imersão ao Flow, uma vez que durante o jogo, o estudante estabelece uma relação entre o nível de dificuldade e o nível de prazer. Para o autor, o jogo é uma das formas mais comuns de se executar atividades lúdicas. Suas regras definem quais são os estímulos relevantes e exclui as distrações, fornecendo elementos motivacionais e incentivos. O jogo proporciona condições que transportam as pessoas a um estado mental que permite a alta concentração, bem-estar, felicidade, criatividade e, consequentemente, um alto desempenho. Esses fatores são fundamentais para o crescimento, e conquista do senso de autodeterminação.

Os jogos tornam-se recursos importantes para o ensino e a aprendizagem em diversas áreas de conhecimento, particularmente para a lógica e para o conhecimento matemático. Eles possibilitam a interação, o desenvolve do raciocínio, da inteligência, imaginação e criatividade, ao mesmo tempo em que envolve as pessoas em atividades lúdicas. Isto posto, observa-se que alguns pesquisadores têm se debruçado sobre estudos que envolvem os jogos em geral, os jogos digitais e ainda os processos de gamificação na área educacional. (Paiva; Tori, 2017; Uribe, 2012; Mendonça Júnior et al., 2013).

Quando são utilizados na educação transformam-se em excelentes recursos pedagógicos possibilitando a construção de conhecimento, por meio do ensinamento de novos conceitos e auxiliando no reforço de conteúdos já adquiridos (Grando, 2000).

Os jogos têm objetivos, regras e metas a serem alcançadas e, de fato, são brincadeiras dirigidas e, particularmente, se configuram como sendo um elemento valioso no desenvolvimento educacional, permitindo que os jogadores/aprendizes experimentem o estado de Flow atingindo a motivação e estimulando a criatividade e aprendizagem por meio de total imersão. 
Nesse sentido, o jogo no contexto educacional pode ser configurado como um instrumento para aprimorar as habilidades necessárias para a construção do saber matemático e no desenvolvimento da autoeficácia, do senso crítico, a argumentação, o espírito investigador e na compreensão de conceitos relacionados ao ensino da matemática e da lógica.

Segundo Csikszentmihalyi (1999), o Flow defini um estado psicológico de desenvolvimento de atividades que são agradáveis a ponto de se perder a noção do tempo, devido à concentração total; possibilitando um estado de "experiência ideal"; a fusão de ação e consciência; experimentando o positivo fora do fluxo, uma vez que esse estado é replicado para outras experiências.

Por outro lado, os estudos apontam que a cognição numérica é influenciada por fatores biológicos, cognitivos e culturais (Santos, et al., 2012); a aprendizagem da matemática abarca mecanismos metacognitivos, a autoeficácia e a autorregulação (Barbosa, 2015). Outras pesquisas apontam que existe relações recíprocas entre autoconceito, autoeficácia, crenças, ansiedade matemática e desempenho em matemática. (Gunderson et al., 2018; Ahmed, 2012; Meece; Wigfield; Eccles, 1990).

Os domínios afetivos e cognitivos estão associados a aprendizagem da matemática. Esses aspectos estão relacionados ao campo da Psicologia Educacional, no qual se destaca os trabalhos de Brito $(1998,1996)$ que tem por objetivo estudar as atitudes e crença de autoeficácia na resolução de problemas matemáticos, dois fatores que podem influenciar no desempenho da matemática.

$\mathrm{Na}$ literatura sobre esses domínios, destacam-se os estudos de Bandura $(1977,1994)$, como já vimos, que afirma que os procedimentos psicológicos, independentemente da sua forma, alteram o nível e força da autoeficácia que é a convicção sobre a própria capacidade em resolver atividades e problemas que envolvam à matemática.

Isto posto, os jogos podem favorecer o desenvolvimento da motivação, autoeficácia, autoestima, autopercepção, emoções, cog- 
nição e afeto, que são variáveis que têm o potencial de identificar e otimizar o processo de aprendizagem, levando em conta o ritmo, e diferenças individuais do estudante.

\section{Considerações Finais}

Foi possível observar que para aprender matemática é necessário que as atividades ou os jogos a serem utilizados, apresentem um equilíbrio entre o desafio e a habilidade da tarefa, elementos que podem ser observados autoeficácia e, que consequentemente, interferem no desempenho do estudante de matemática.

A noção de estar no controle é central para a experiência do Flow assim como para a autoeficácia. A aprendizagem da matemática exige ordem sequencial sobre o conteúdo a ser aprendido. Para executar uma atividade, o aprendiz precisa ter domínio de cada etapa do desenvolvimento do tema abordado, pois só assim, será possível ir adiante no desenvolvimento do raciocínio.

Ao elaborar uma atividades educacionais, é necessário pensar não só nas questões cognitivas e intelectuais, mas também nas questões afetivas, emocionais e motivacionais. A Metodologia Ativa permite uma reflexão acerca desses fatores, bem como permite levar em consideração os elementos do Flow como: metas e objetivos claros; feedback imediato sobre o progresso que está se realizando; equilíbrio entre a ação e a capacidade de realizá-la; sensação de controle sobre a atividade; concentração profunda; foco temporal; fim da noção de tempo real; perda da autoconsciência reflexiva e atividade gratificante; e, consequentemente, refletir como estas condições promovem o desenvolvimento da autoestima, autoconceito e autoeficácia.

Como limitação desse estudo, observamos a necessidade de uma análise mais profunda acerca das teorias e a busca por artigos já publicados que abordem essa temática. Este ensaio teórico sugere novas investigações acerca da correlação entre a Teoria do Flow e a Teoria Cognitiva Social no processo de aprendizagem da matemática, com vistas a categorizar e identificar o momento ideal em que o aprendiz atinge o estado de Flow e amplia suas concepções 
acerca da autoeficácia. Como proposta, o desenvolvimento de um instrumento psicométrico validado para as duas teorias.

\section{Referências}

ANDRADE, P. E.; PRADO, P. S. T.; CARMO, J. S. Das Representações Numéricas Inatas à Matemática Culturalmente Construída. In: Trends in Psychology, v. 23, no 1, p. 225-242, 2015.

ACELAJADO, M. J. The impact of using technology on students' achievement, attitude, and anxiety in mathematics. In Proceedings of the 10th International Congress on Mathematical Education (ICME-10), Topic Study Group: The Role and Use of Technology in the Teacbing and Learning of Mathematics. Copenhagen: Denmark, July 4-11, 2004.

BANDURA. A. Self-efficacy: toward a unifying theory of behavioral change. In: Psychological Review, v. 84, n. 2, p. 191-215, 1977.

. Social cognitive theory: An agentic perspective. In: Asian Journal of Social Psychology, v. 2, p. 21-41, 1999.

. Self-efficacy. In: RAMACHAUDRAN, V. S. (Ed.) Encyclopedia of human behavior. Cambridge: Academic Press, v. 4. p. 71-81, 1994.

BARBOSA, D. C. B. P. Intervenção neuropsicológica para manejo da ansiedade matemática e desenvolvimento de estratégias metacognitivas. Dissertação de Mestrado em Neurociências. Universidade Federal de Minas Gerais, UFMG, 2015.

BRITO, M. R. F (1998). Adaptação e validação de uma escala de atitudes em relação à matemática. In: Zetetike, v. 6(9), p. 45-63, 1998.

BRITO, M.R.F. Um estudo sobre as atitudes em relação à matemática em estudantes de $1^{\circ}$ e $2^{\circ}$ graus. Livre Docência na área de Aprendizagem do Departamento de Psicologia Educacional Campinas, SP: Faculdade de Educação da UNICAMP, 1996.

CSIKSZENTMIHALYI, M. Flow: the psychology of optimal experience. 1st ed. Harper Perennial Modern Classiscs, 1990.

A descoberta do fluxo: a psicologia do envolvimento com a vida cotidiana. Rio de Janeiro. Rocco, 1999. 
The flow experience and its significance for buman psychology. In: CSIKSZENTMIHALYI, Mihaly. Optimal experience: psychological studies of flow in consciousness, Cambridge, UK: Cambridge University Press, 1988.

Play and Intrinsic Rewards. In: CSIKSZENTMIHALYI, M. Flow and the Foundations of Positive Psychology: The Collected Works of Mihaly Csikszentmibalyi. Springer Science. Business Media, Dordrecht, p. 135-153, 2014.

CSIKSZENTMIHALYI, M.; NAKAMURA, J. The Concept of Flow. In: SNYDER, C.; LOPEZ, Shane. The Handbook of Positive Psychology. New York: Oxford University Press, 2002.

GEIST, E. (2010). The anti-anxiety curriculum: Combating mathematics anxiety in the classroom. Journal of Instructional Psychology, 37(1), 24-31GUNDERSON, E. A.; PARK, D.; MALONEY, E. A.; BEILOCK, S. L.; LEVINE, S. C. Reciprocal relations among motivational frameworks, math anxiety, and math achievement in early elementary School. In: Journal of Cognition and Development, v. 19, n.1, p. 21-46, 2018.

GOURGEY, A. The Relationship of Misconceptions about Math and Mathematical Self-Concept to Math Anxiety and Statistics Performance. In: Annual Meeting of the American Educational Research Association, New Orleans, 1984.

GRANDO, R. C. O conhecimento matemático e o uso de jogos na sala de aula. 239 fs. Tese de doutorado em educação. Universidade Estadual de Campinas, 2000.

HACKETT, G.; BETZ, N. E. An exploration of the mathematics self-efficacy/mathematics performance correspondence. In: Journal for Research of Mathematics Education, Vol. 20, No. 3, 1989. pp 261-273.

MENDONÇA JÚNIOR, M. M; BARBALHO, L. M.; PEGADO, V.; PINHEIRO, L. Gaminception: ferramenta de ludificação no processo de design de jogos. In: Proceedings of SBGames, p. 181-188, 2013.

MEECE, J.L.; WIGFIELD, A.; ECCLES, J. S. Predictors of math anxiety and its influence on young adolescents' course enrollment intentions and performance in mathematics. In: Journal of Educational Psychology, v. 82 (1), p. 60-70, feb., 1990.

PAIVA, C. A.; TORI, R. Jogos Digitais no Ensino: Processos cognitivos, benefícios e desafios. XVI Proceedings of SBGames. Curitiba, p. 1052-1055, nov, 2017.

PAVANELO, E; LIMA, R. Sala de aula invertida: a análise de uma experiência na disciplina de Cálculo I. In: Bolema: Boletim de Educação Matemática, v. 31 n. 58, Aug. Rio Claro: UNESP, 2017. 
SANTOS, F. H.; SILVA, P. A.; RIBEIRO, F. S.; DIAS, A. L. R. P.; FRIGÉRIO, M. C.; DELLATOLAS, G.; ASTER, M. V. Number processing and calculation in Brazilian Children Aged 7-12 years. In: The Spanish Journal of Psychology, v. 15. N. 2. Online Firt, 2012.

SCARPELLO, G. Helping students get past math anxiety. In: Techniques: Connecting Education and Careers, 82(6), 34-35, 2007.

STUART, V. B. Math curse or math anxiety? Teaching Children Mathematics, 6(5), 330, 2000.

VALENTE, J.A. A telepresença na formação de professores da área de Informática em Educação: implantando o construcionismo contextualizado. Actas do IV Congresso Ibero-Americano de Informática na Educação. RIBIE98, Brasília, CD-ROM/trabalhos/232.pdt, 1998. Disponível em: <http://lsm.dei.uc.pt/ribie/ docfiles/txt200342421644232.PDF>. Acesso em: agosto de 2014.

A comunicação e a educação baseada no uso das tecnologias digitais de informação e comunicação. Revista UNIFESO: Humanas e Sociais, v. 1, n. 1, p. 141-166, 2014. Disponível em: <http:revistaunifeso.filoinfo.net/index.php/revistaunifesohumanasesociais/article/view/17/24>.Acesso em: 05 jul. 2016. 